\title{
Hydrodynamic behavior of bubbles at gas-evolving electrode in ultrasonic field during water electrolysis
}

\author{
Kyung Min Cho, P.R. Deshmukh, Weon Gyu Shin " \\ Department of Mechanical Engineering, Chungnam National University, Daejeon 34134, Republic of Korea
}

\section{A R T I C L E I N F O}

\section{Keywords:}

Sonoelectrochemistry

Ultrasound

Bubble resistance

Hydrogen evolution reaction

Bubble dynamics

Alkaline water electrolysis

\begin{abstract}
A B S T R A C T
In electrochemical processes, gas bubbles on the electrode can cause an increase in both overpotential and ohmic voltage drop which leads to higher energy consumption. Applying power ultrasound during water electrolysis can help to reduce the overpotential, enhance mass transfer, and save energy. In this study, we investigated the effect of ultrasound $(20 \mathrm{kHz})$ on the hydrogen evolution reaction (HER) on a stainless steel plate with varying concentrations of $\mathrm{NaOH}$ solutions at $298 \mathrm{~K}$, using linear sweep voltammetry (LSV). We especially focused on understanding the bubble behavior on the stainless steel plate during HER using high-speed imaging in ultrasonic field. When ultrasound was applied to solutions with $\mathrm{NaOH}$ concentrations of $0.1,0.5,1 \mathrm{M}$, the current density increased by about 9.0, 5.9, $2.8 \%$, respectively. As the ultrasound irradiation began, the bubbles tended to hover around on the electrode surface, coalescing with other bubbles, rather than rising. When the size of the coalesced bubbles became too large to stay on the surface of the electrode, they were expelled from the ultrasonic field. The repeated collapse and coalescence of these bubbles was observed while they were rising. The velocity increased about 2 times when ultrasound irradiation began, and increased by more than 6 times in the ultrasonic field. More nucleation of bubbles was observed on the electrode in the ultrasonic field. Using ultrasound reduced the critical diameter of bubbles which detached from the electrode, from 58.0 to $15.9 \mu \mathrm{m}$, and the residence time of the bubbles, from 533 to $118 \mathrm{~ms}$. Further, when the ultrasound was applied, the mean diameter of bubbles decreased from 71.8 to $17 \mu \mathrm{m}$. Hence, bubble coverage on the electrode surface decreased from 8.3 to $1 \%$ despite an increase in the total number of bubbles. As a result, ultrasound was found to be effective for hydrogen production during water electrolysis, increasing current by the faster removal of gas from the stainless steel plate.
\end{abstract}

\section{Introduction}

Hydrogen is considered the most suitable alternative to conventional energy sources. Using hydrogen as a fuel has many benefits; it is efficient, environmentally friendly, non-toxic, and renewable [1-3]. Hydrogen fuel has a very high specific energy compared to other conventional fuels and emits a very low level of greenhouse gases when burnt. Hydrogen can be produced wherever water exists using water electrolysis, making it a clean and sustainable energy source. Electrolysis is an electrochemical process of decomposing ionic compounds into their fundamental elements by passing electricity through two electrodes in an aqueous solution containing ions [4]. Currently, water electrolysis accounts for about $4 \%$ of total hydrogen production worldwide [5]. However, making water electrolysis cost-effective is still a challenge. Alkaline water electrolysis (AEC) using common metals and alkaline solutions is low-cost, and can be readily scaled up. The key challenge with alkaline water electrolysis is to increase current density and lower energy consumption.

There are two half-reactions in alkaline water electrolysis: the hydrogen evolution reaction (HER) at the cathode and the oxygen evolution reaction (OER) at the anode. Two half-reactions are expressed as [6]:

HER : $4 \mathrm{H}_{2} \mathrm{O}(\mathrm{l})+4 e^{-} \rightarrow 2 \mathrm{H}_{2}(\mathrm{~g})+4 \mathrm{OH}^{-}(a q)$

OER : $4 \mathrm{OH}^{-}(a q) \rightarrow \mathrm{O}_{2}(g)+2 \mathrm{H}_{2} \mathrm{O}(l)+4 e^{-}$

$\mathrm{H} 2$ and $\mathrm{O} 2$ bubbles in both HER and OER cause electrochemical losses during water splitting. Thus, in real water splitting, the cell voltage is higher than the decomposition voltage. The total cell voltage is shown in Eq. (3) [6]

$V_{\text {cell }}=\left|E_{c}-E_{a}\right|+j \times \sum R=E^{\text {rev }}+\left|\eta_{a}\right|+\left|\eta_{c}\right|+\mathrm{j} \times \sum R$

\footnotetext{
* Corresponding author.

E-mail address: wgshin@cnu.ac.kr (W.G. Shin).
} 
where $E_{a}$ is the anode potential for OER, $E_{c}$ is the cathode potential for HER, $\mathrm{j}$ is the current density, $\sum R$ is the total ohmic resistance, $E^{\text {rev }}$ is the reversible potential (Nernst), $\eta_{a}$ is the anode overpotential, and $\eta_{c}$ is the cathode overpotential. Based upon Eq. (3), the total cell voltage is influenced by the theoretical decomposition voltage, overpotentials, and ohmic voltage drop. The total ohmic resistance is shown in Eq. (4). [6]

$\sum R=R_{e}+R_{m}+R_{b}+R_{c}$

where $\mathrm{Re}$ is the electrolyte resistance, $\mathrm{Rm}$ is the membrane resistance, $\mathrm{Rb}$ is the bubble resistance, and $\mathrm{Rc}$ is the circuit resistance. Although Rc and Rm are constant and can be decreased by optimizing the membrane and wire connection, $\mathrm{Rb}$ is not constant. The bubbles attached to the electrode surface disturb the electric field and lead to a high bubble resistance $\mathrm{Rb}$ which causes a high ohmic voltage drop [7]. In addition, the dispersion of these bubbles in the electrolyte solution increases the electrolyte resistance Re by lowering the electrolyte conductivity. As the number of bubbles generated at the electrode increases, the bubble resistance increases, resulting in more energy consumption. Thus, minimizing the gas volume and bubble residence time at the electrode is important for keeping high current densities in water electrolysis.

The anode and cathode overpotentials can be reduced by operating the cell at higher temperatures $\left(65-80^{\circ} \mathrm{C}\right)$ [8]. Using an effective electrocatalytic material as an electrode is another way to reduce the overpotentials. Xu et al. [9] used superwetting electrodes coated with superaerophobic film for water electrolysis. Chen et al. [10] used a grooved anode to allow the bubbles to escape more quickly. Tiwari et al. [11] reported a 'bubble-free' electrolyzer, with electrodes comprised of Gortex gas diffusion layers coated with catalyst. Also, techniques have been studied to reduce the overpotential by enhancing the bubble detachment from the electrode surface using external sources. Bakker et al. [12] used pressure swings to remove bubbles on the electrode in alkaline water electrolysis. Koza et al. [13] showed that the bubble separation process is significantly influenced by MagneticHydrodynamic field (MHD). Wang et al. [14] verified that the critical radius of the bubbles in HER is smaller in a super gravity field, and bubbles disengage faster from the electrode with a larger buoyancy force.

Ultrasound has been used as a potential method for efficient bubble removal in hydrogen production. High intensity low-frequency ultrasound (20-100 kHz) based on acoustic cavitation is an eco-friendly method for efficient water electrolysis hydrogen production [15]. The effect of ultrasound on water electrolysis was initially reported by Moriguchi in the 1930 s [16]. Sheng-De Li and Richard reported increased energy efficiency during the electrolysis process with ultrasound $[17,18]$. Recently, a few studies by Pollet and his research group have focused on applying ultrasound for hydrogen production. Pollet introduced the use of power ultrasound and provided comprehensive information about sonochemical and sonoelectrochemical processes [19]. Zadeh and Symes showed that the overall overpotential of electrolysis using different concentrations of weak acidic (H2SO4) and alkaline solutions $(\mathrm{NaOH}, \mathrm{KOH})$ was reduced by ultrasound [20,21]. Pollet et al. have investigated the effect of ultrasonic intensity, ultrasonic frequency, and electrode materials on the electrochemical processes with different electrode materials. For example; the effect of ultrasonic frequency and intensity on electrode kinetic parameters [22], effect of ultrasound on the oxidation of thiosulphate using stainless steel and platinum electrodes [23], and reduction study of silver thiosulphate in the absence and presence of ultrasound [24]. Islam et al. [25] showed that energy consumption in electrolysis can be reduced by using ultrasound.

The ultrasonic effect produced by acoustic cavitation in solution generates new reaction mechanisms in the electrochemical process [26] such as:
1. Degassing of the solution and electrode surface.

2. The disruption of the Nernst diffusion layer.

3. Activation and cleaning of the electrode surface.

4. Enhancing mass transfer between near surfaces and the bulk solution.

By exploiting these mechanisms, Islam et al. [25] showed that power ultrasound has many advantages for overcoming the limitations of water electrolysis. Up to now, there have only been a few studies about hydrogen production using a sonoelectrochemical process. Furthermore, they have only focused on the effect of power ultrasound on the current density and hydrogen production efficiency, not bubbles. The bubble behavior at the gas-evolving electrode in an ultrasonic field (gas bubble removal process) is still not clear.

In this study, we investigated how bubble behavior on a stainless steel plate electrode in HER was affected when ultrasound was applied. The current density of HER on a stainless steel plate electrode under both silent condition and sonication with varying $\mathrm{NaOH}$ concentrations was measured using linear sweep voltammetry (LSV). Using high-speed imaging, we measured the diameter and the velocity of bubbles in HER under both silent condition and sonication. Further, we correlated the hydrodynamic behavior of hydrogen bubbles to the fast gas removal mechanism when the ultrasound was applied.

\section{Experimental methods}

\subsection{Sonoelectrochemical cell design and flow system}

Our experiments were performed in a double-jacketed reactor (Fig. 1 (a)). This double-jacketed reactor, called a Besançon cell, prevents electrolyte contamination due to erosion of the ultrasonic probe tip and makes it easier to control the electrolyte temperature $[27,28]$. The inner dimension of the reactor was $50 \times 50 \times 65 \mathrm{~mm}$ (width $\times$ depth $\times$ height). The working volume of the inner cell was $20 \mathrm{ml}$. The crosssection of the jacket (10 mm thick plexiglass) and the inner cell (2 $\mathrm{mm}$ thick glass) were designed flat to minimize optical distortion. The reactor was equipped with a single horn type ultrasonic transducer (ULH-700S, Ulsso Co., Korea) operating at $20 \mathrm{kHz}$ (700 W). A $10 \mathrm{~mm}$ diameter ultrasonic horn (Ti Alloy) was used as a sonotrode. The ultrasonic horn was immersed $20 \mathrm{~mm}$ through the base of the reactor using an O-ring. The distance from the bottom of the inner cell to the ultrasonic horn tip was $10 \mathrm{~mm}$. A solution of distilled water with $\mathrm{NaOH}$ (Extra Pure GRADE, DUKSAN PURE CHEMICALS CO., LTD., Korea) was used as the electrolyte. Distilled water was used as a cooling fluid in the flow system. A stainless steel plate (AISI 304, $100 \mathrm{~mm}^{2}$ electrode area) was used as a working electrode (WE) and a platinum wire $(20 \mathrm{~mm}$ immersion length with the diameter of $0.5 \mathrm{~mm}$ ) was used as a counter electrode (CE). $\mathrm{An} \mathrm{Ag} / \mathrm{AgCl}$ electrode was used as the reference electrode (RE). The distance between the center of the working electrode and the counter electrode was $10 \mathrm{~mm}$. All the electrodes were placed 5 $\mathrm{mm}$ above the bottom of the inner cell.

The flow system of the sonoelectrochemical process is illustrated in Fig. 1(b). The cooling fluid was circulated inside the jacket during sonication. The temperature of the circulating fluid can be increased by sonication. Thus, to maintain the temperature of the electrolyte inside the inner cell, the circulating fluid needs to be cooled without disturbing the sonication which can cause fluctuations. The flow rate of the cooling fluid was set to $0.5 \mathrm{lpm}$. Also, a heat exchanger consisting of thermoelectric modules was used as a water cooler. The heat exchanger was operated by a temperature controller which monitored the temperature of the circulating fluid in the flow system. The temperature of the liquid inside the inner cell and the circulation tank was recorded by a data logging thermometer (Oakton Temp-300, Oakton, USA) using K-type thermocouple probes. During sonication, the temperature of the electrolyte solution was determined by adjusting the temperature of the circulating fluid. The temperature controller was set to maintain the 


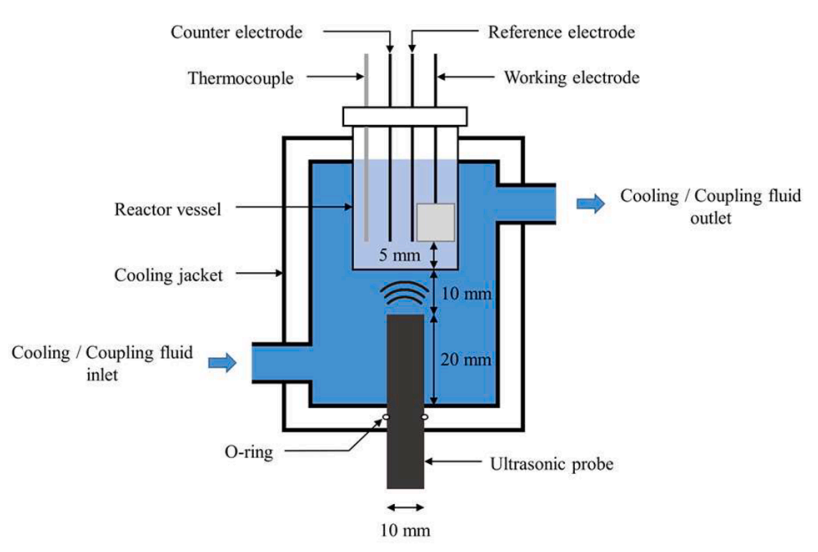

(a)

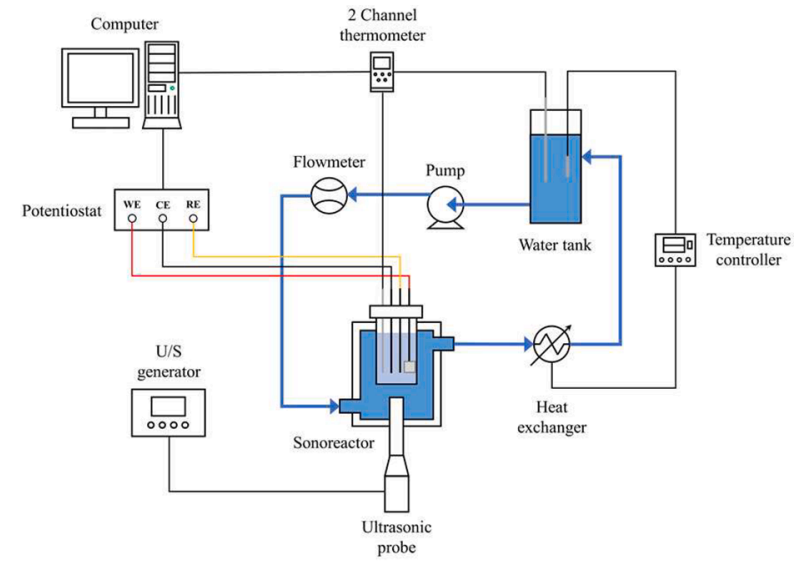

(b)

Fig. 1. Schematic diagram of experimental set-up for sonoelectrochemical process :(a)double-jacketed cell (Besançon cell), (b) flow system.

temperature of the electrolyte solution at $298 \mathrm{~K}$ throughout the experiments. The current of the sonoelectrochemical cell was measured by a potentiostat (Vertex.1A, Ivium tech., Netherlands) controlled by Ivium software. Linear sweep voltammetry (LSV) was utilized to evaluate the effects of ultrasound in this study.

\subsection{Bubble observation system}

In this study, we observed HER on the surface of the electrode to investigate the effect of ultrasound in water electrolysis. Fig. 2 illustrates the experimental set-up for the high-speed imaging. A high-speed video camera (pco.dimax HS1, PCO Image, Germany) with an optical microscope (OPTEM Zoom 70XL, Excelitas tech., USA) and a light source with LED were used for the images of the bubbles. Images were recorded at 5,000 frames per second for $2 \mathrm{~s}$. In addition, a CCD camera (DFK 23G445, Imagingsource, Germany) with a SLR lens (SIGMA 24-70 mm f/2.8 EX DG, SIGMA Co., Japan) was used for the high definition color image of the electrode surface.

\subsection{Acoustic power measurement}

Acoustic power $\left(P_{w}\right)$ or intensity $(\psi)$ is an important factor that dominates the reactions in the sonoelectrochemical process. During sonication, the temperature of the liquid inside the inner cell increases as the mechanical energy is converted to heat. The transmitted acoustic power was measured by sonicating $10 \mathrm{ml}$ ultrapure water in the inner cell for $60 \mathrm{~s}$. During sonication, both the inlet and the outlet of the sonoreactor were closed to measure the acoustic power, and the temperature of the ultrapure water was recorded every second by a data logging thermometer [28]. Using a standard calorimetric method presented by Mason [29] and Contamine [30], the acoustic power can be calculated using Eq. (5) as:

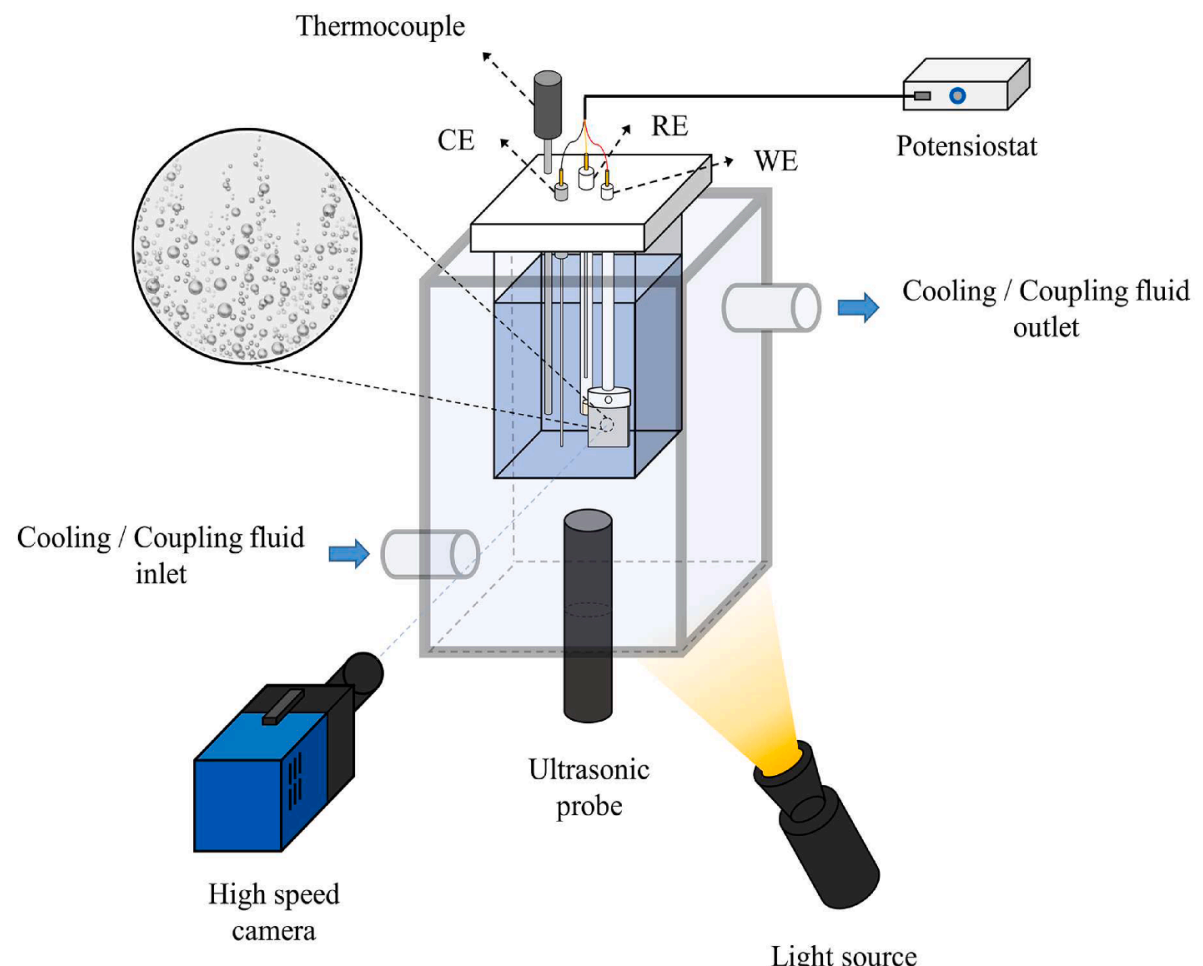

Fig. 2. Schematic diagram of experimental set-up for high-speed imaging. 
$P_{t}=m C_{p} \frac{d T}{d t}$

where $P_{t}$ is the transmitted acoustic power from the coupling fluid to the reactor in $\mathrm{W}, m$ is the mass of the liquid in $\mathrm{g}, C_{p}$ is the specific heat capacity of the liquid and $\frac{d T}{d t}$ is the temperature slope per time in $\mathrm{K} \mathrm{s}^{-1}$. The transmitted acoustic power was calculated using the calorimetric method with a specific heat of the water as $4.186 \mathrm{~J} / \mathrm{g} \mathrm{K}$ at $298 \mathrm{~K}$ and $101.325 \mathrm{kPa}$. Fig. 3 shows the transmitted acoustic intensity ( $\psi$ in $\mathrm{W} /$ $\left.\mathrm{cm}^{2}\right)$ in which the transmitted acoustic power $\left(P_{t}\right.$ in $\left.\mathrm{W}\right)$ is divided by the area of the ultrasonic probe tip $\left(A_{U S}\right.$ tip in $\mathrm{cm}^{2}$ ) for each ultrasonic amplitude.

\section{Results and discussion}

\subsection{Effect of ultrasound on HER performance}

Fig. 4 shows the linear sweep voltammetry (LSV) curves for the HER on stainless steel under silent condition and sonication. LSV was performed in the $0.1,0.5$, and $1.0 \mathrm{M} \mathrm{NaOH}$ electrolyte concentrations at a scan rate of $2 \mathrm{mV} / \mathrm{s}$. All the potential was converted into the reversible hydrogen electrode (RHE) using Eq. (6) as:

$\mathrm{E}_{\mathrm{RHE}}=\mathrm{E}_{\mathrm{Ag} / \mathrm{AgCl}}+\mathrm{E}_{\mathrm{Ag} / \mathrm{AgCl}}^{0}+0.059 \mathrm{pH}$

where $\mathrm{E}_{\mathrm{Ag} / \mathrm{AgCl}}^{0}$

$=0.1976 \mathrm{~V}$ at $250 \mathrm{C}$ and $\mathrm{pH}$ of $\mathrm{NaOH}$ electrolyte $=13$.

For sonication, an $80 \%$ ultrasonic amplitude was used. Under silent condition, the current densities of the stainless steel electrode were 48.2, 108.9 , and $144 \mathrm{~mA} / \mathrm{cm}^{2}$ in the $0.1,0.5$, and $1 \mathrm{M} \mathrm{NaOH}$ electrolytes, respectively, when the applied potential was $-1.0 \mathrm{~V}$ vs. RHE. Under sonication with an applied potential of $-1.0 \mathrm{~V}$ vs. RHE, the SS electrode exhibited current densities of 52.5, 115.3, and $148.1 \mathrm{~mA} / \mathrm{cm}^{2}$ in the 0.1 , 0.5 , and $1 \mathrm{M} \mathrm{NaOH}$ electrolytes, respectively. By comparing the current densities under the silent condition and sonication, we observed the current density was enhanced under sonication. There were 9.0, 5.9, and $2.8 \%$, increases in current density, when the $\mathrm{NaOH}$ electrolytes were $0.1,0.5$ and $1 \mathrm{M}$, respectively. As noted, the enhancement in current density under the ultrasonic field decreased as the electrolyte concentration increased. This is because the resistance of the electrolyte was lowered by the higher electrolyte concentration [17].

To maximize the ultrasound effect, the Pollet research group used face-on geometry, placing a Pt disc electrode vertically to the ultrasonic irradiation direction [31]. In this study, we used side-on geometry to

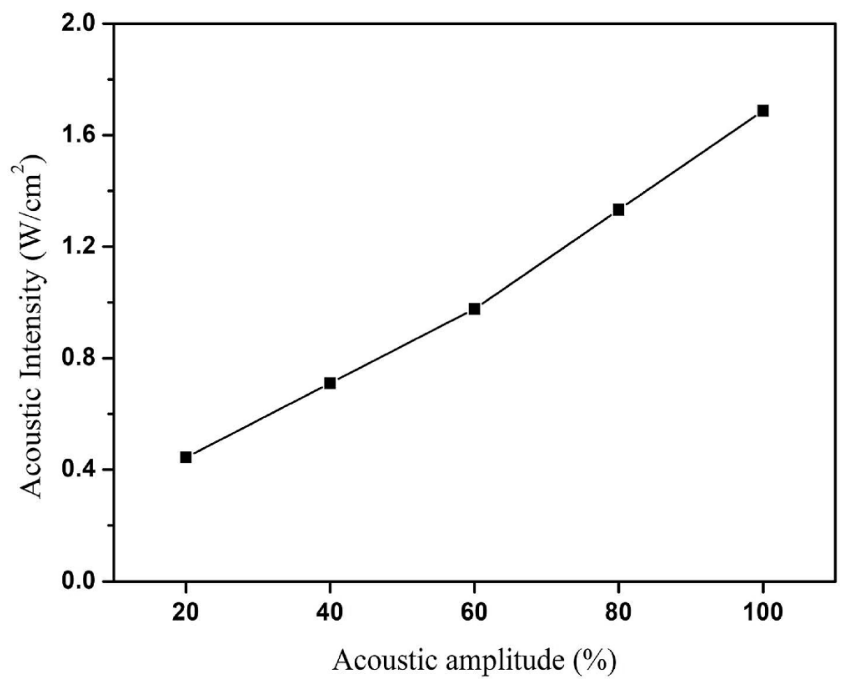

Fig. 3. The transmitted acoustic power from the coupling fluid to the inner cell at different acoustic amplitudes.

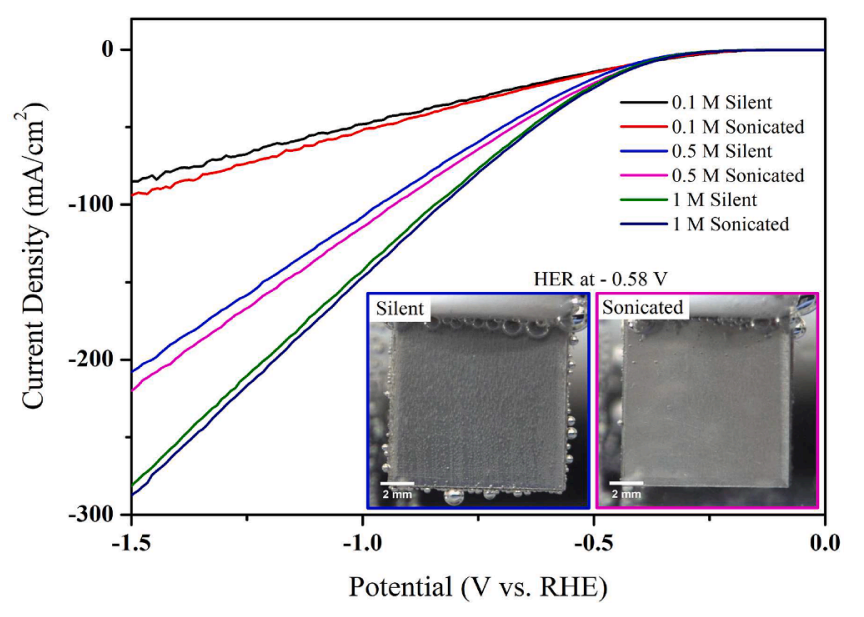

Fig. 4. Linear sweep voltammetry (LSV) of HER on a stainless steel electrode under silent condition and sonication for different $\mathrm{NaOH}$ concentrations at 298 $\mathrm{K}$ and at a scan rate of $2 \mathrm{mV} / \mathrm{s}$.

observe the bubbles on the electrode.

\subsection{Bubble dynamics in ultrasonic field}

\subsubsection{Bubble coalescence induced by ultrasound}

When bubbles are subjected to ultrasound, the bubbles begin to oscillate and then interact with the ultrasonic field, inducing the primary Bjerknes force $[32,33]$. Then the small bubbles start to move toward the larger bubbles, influenced by the secondary Bjerknes force [34]. This force is induced by the difference in compressibility and density between bubbles. Fig. 5 presents front view snapshots of the bubbles during the HER on a stainless steel electrode immediately after ultrasound irradiation. When ultrasound irradiation is initiated, the hydrogen bubbles start to oscillate (Fig. 5(a)) and then agglomerate (Fig. 5(b)-(i)) or coalesce (Fig. 5(j)) due to the radiation force. Coalescence is the merging of two or more bubbles into one bubble and agglomeration is the clustering of two or more bubbles without merging. In this process (Fig. 5(b)-(i)), small bubbles agglomerates around the nearby larger bubble and form a bubble cluster [35]. After agglomeration, bubbles coalesced due to unstable film caused by oscillation and collision. The buoyancy force increases as the bubble size increases due to coalescence. As the bubbles grew too large to stay on the surface of the electrode, they were expelled from the ultrasonic field (Fig. 5(j)). The coalesced bubbles quickly slided up with agglomerating along the surface of the vertical electrode, not jumping off the surface of the electrode. Those bubbles rose until they reached the top section of the electrode and were eventually detached from the edge of the electrode. After a few seconds of ultrasound irradiation, there were only small bubbles left on the electrode surface.

The effect of ultrasound on the bubble behavior depends on the material and the type of electrode. Bubbles tend to form clusters without merging on a Pt wire surface [31]. On the other hand, the coalescence of the bubbles was observed more frequently on the stainless steel plate surface. The distance among bubbles is small on the Pt electrode surface but large on the stainless steel electrode surface. Also, the surface area of the plate type electrode was larger than that of the wire type electrode. Therefore, it took more time for bubbles on the stainless steel plate to be agglomerated than those on the Pt wire. The collision force of the bubbles on the stainless steel plate was stronger as they had more time and space to be accelerated in ultrasonic field. As a result of their strong collision force, the bubbles on the stainless steel plate were more likely to coalesce. In contrast, bubbles on the Pt wire agglomerated forming bubble clusters because of their weak collision force [36]. In addition, the bubble size on the stainless steel plate was larger than that on the Pt wire. As the surface area of bubbles exposed to ultrasound increased, 


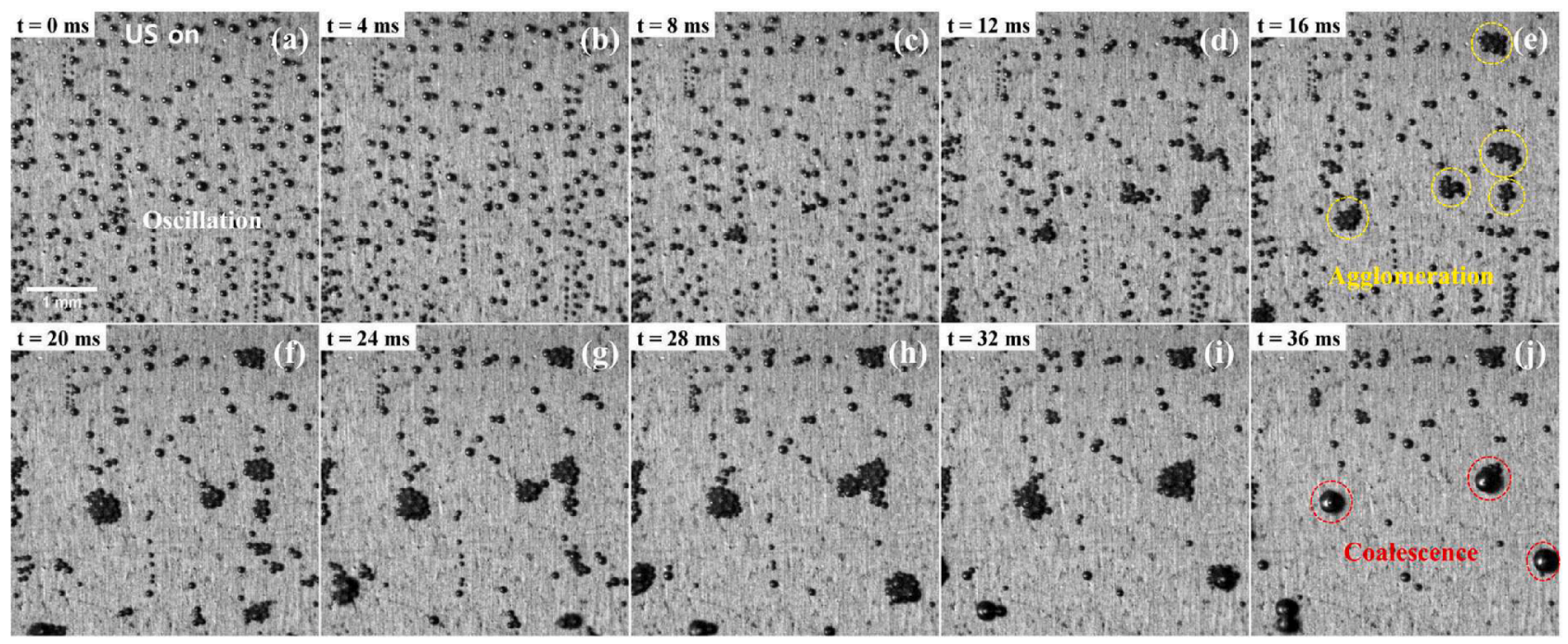

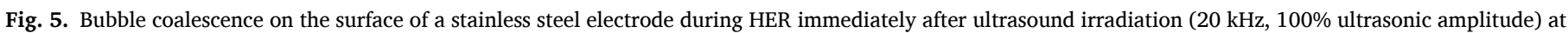
$-0.58 \mathrm{~V}$ vs. RHE in $0.5 \mathrm{M} \mathrm{NaOH}$ at $298 \mathrm{~K}$.

they tended to coalesce due to unstable film.

\subsubsection{Bubble behavior under continuous sonication}

To understand the whole sonoelectrochemical process at a gasevolving electrode in an ultrasonic field, it is important to investigate the bubble behavior. After the previously detached bubbles had coalesced and were expelled by ultrasound irradiation, new bubbles at the nucleation sites were exposed to the ultrasonic field. Fig. 6 shows a schematic illustration of the hydrogen bubble removal mechanism in the ultrasonic field. Mainly, two types of bubble behaviors were observed during continuous sonication: coalescence and dispersion. Under sonication, bubbles on the electrode surface tended to agglomerate with one another first, rather than grow, until they reached the critical size at the nucleation site. Small bubbles generated on the electrode surface even moved downward to coalesce with other bubbles. After leaving the nucleation site, bubbles in the ultrasonic field started to pop up while oscillating on the electrode surface. These small bubbles could easily pop up and slide on the electrode surface because ultrasound had assisted the attached bubbles to overcome the surface tension at the gas-solid interface [37]. As these bubbles grew they began sweeping and merging neighboring smaller bubbles in the ultrasonic field. When the size of the coalesced bubbles became larger, they began to rise due to their increased buoyancy. Tiny bubbles were generated adjacent to the electrode surface during the sonoelectrochemical process. The formation and behavior of the tiny bubbles are explained in the following section.

Fig. 7 illustrates the hydrogen bubble behavior on the surface of a stainless steel electrode under continuous sonication, consisting of

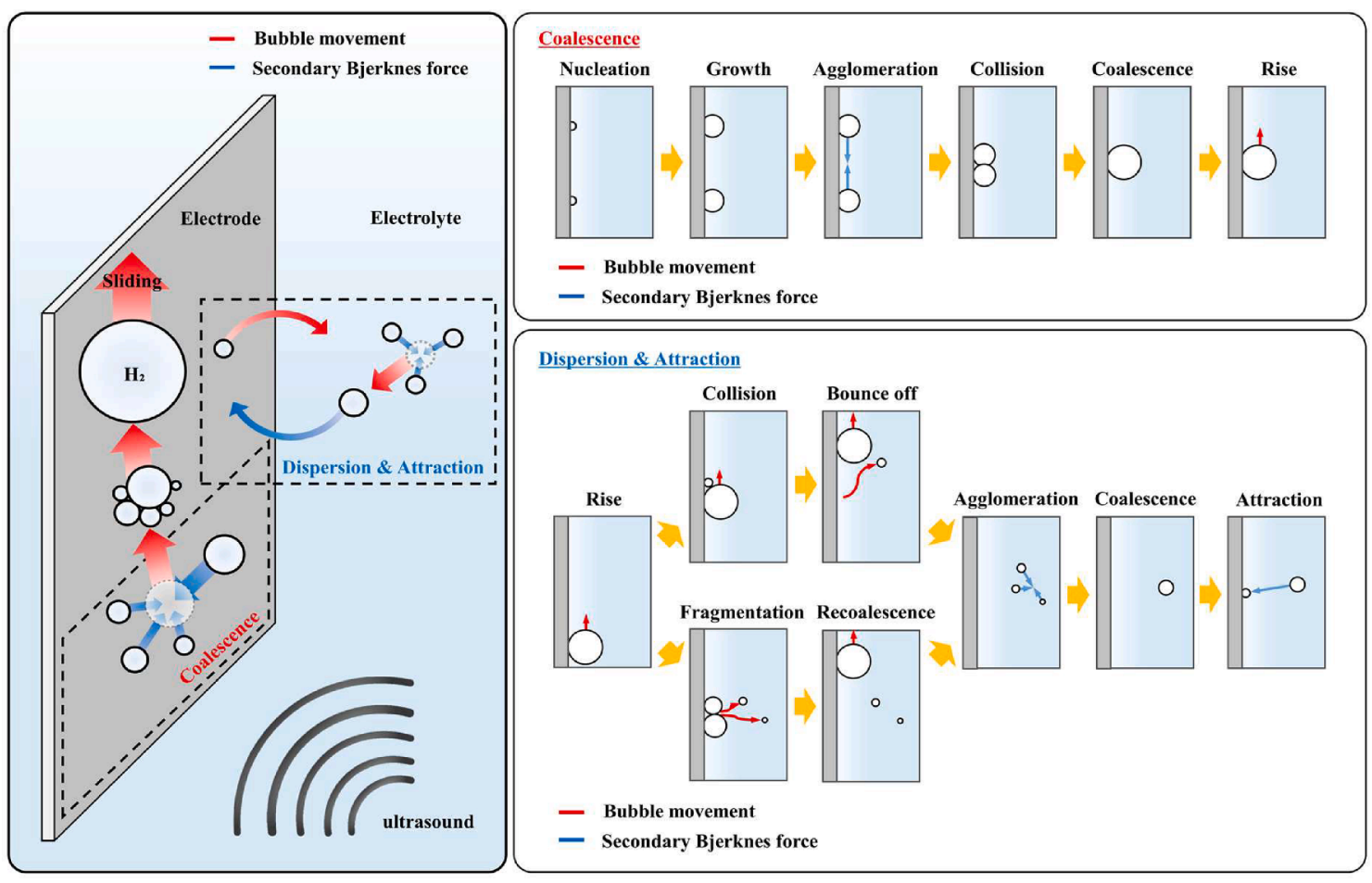

Fig. 6. Schematic illustration of the hydrogen bubble removal mechanism in an ultrasonic field. 
(a)

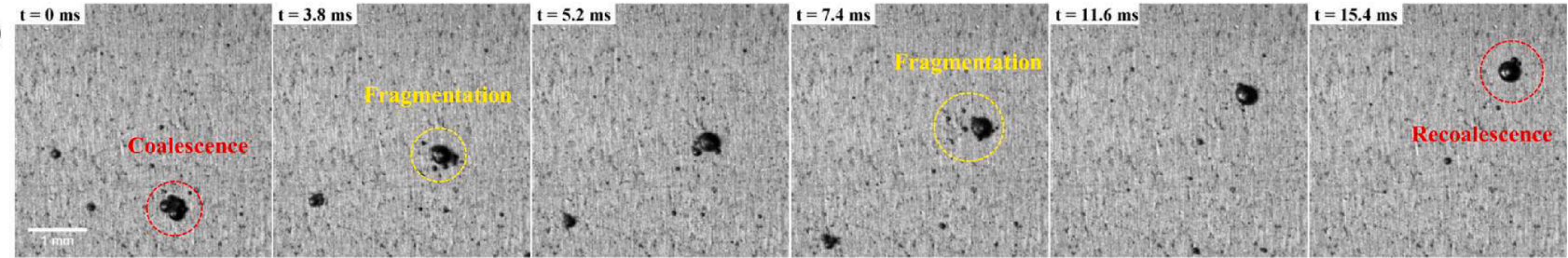

(b)

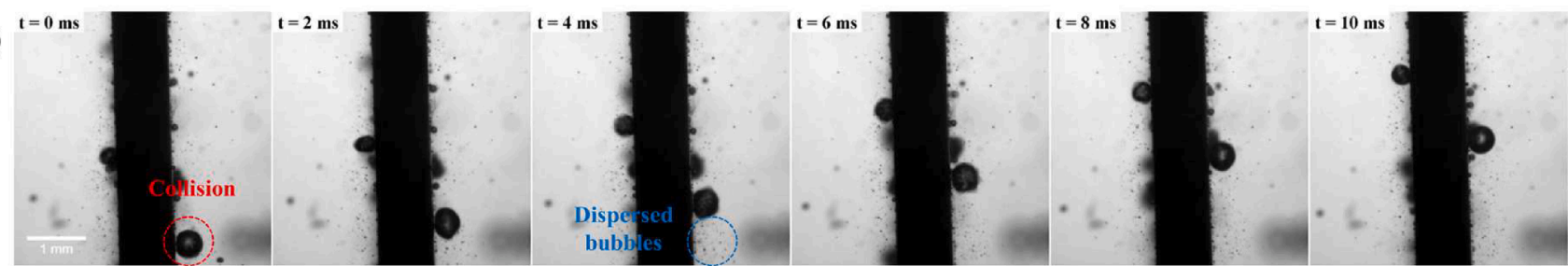

(c)
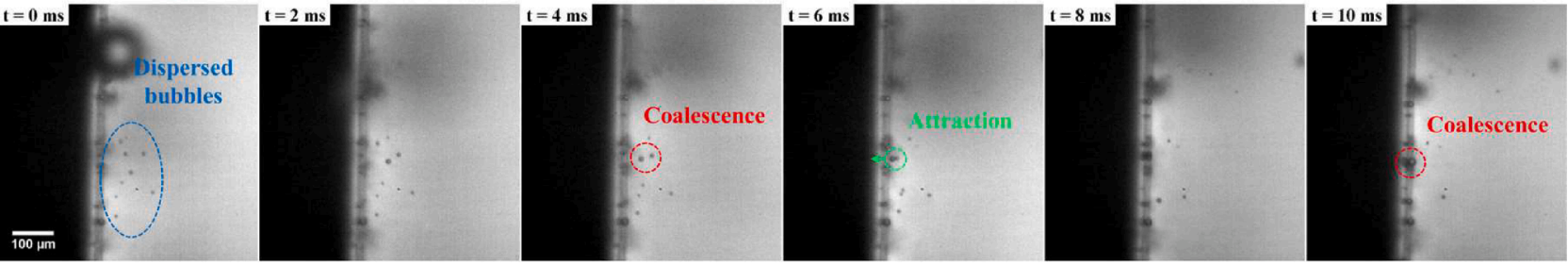

Fig. 7. Behavior of bubbles on the electrode surface during HER under continuous sonication ( $20 \mathrm{kHz}, 100 \%$ ultrasonic amplitude) at $-0.58 \mathrm{~V}$ vs. RHE in $0.5 \mathrm{M}$ $\mathrm{NaOH}$ : (a) fragmentation, (b) collision, and (c) attraction.

fragmentation, collision, and attraction. While rising, the film of the coalesced bubbles is unstable because of continuous oscillation and collisions. Therefore, the coalesced bubbles can be split into smaller bubbles in the ultrasonic field (Fig. 7(a)). These small bubbles, separated from the large bubble, agglomerate and coalesce immediately after fragmentation due to the secondary Bjerknes force. The process of coalescence and fragmentation of bubbles is repeated while rising [38]. The collapse of coalesced bubbles was observed only with small size bubbles.

As the bubble size became larger, fragmentation was no longer observed because of increased surface tension. In this repeated process, some tiny bubbles failed to coalesce again and were separated from the (a)

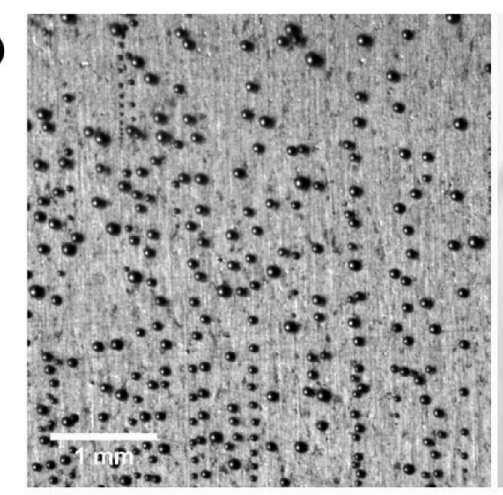

(b)

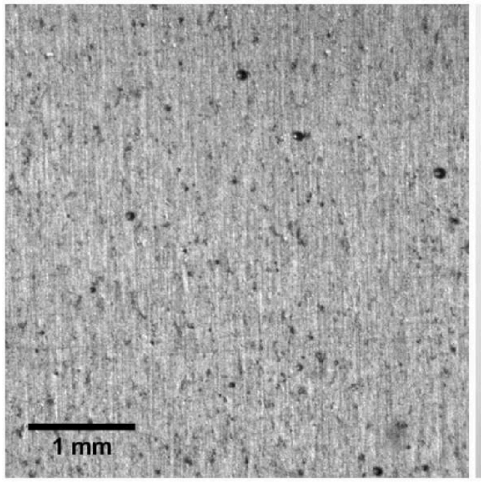

\section{Front view}

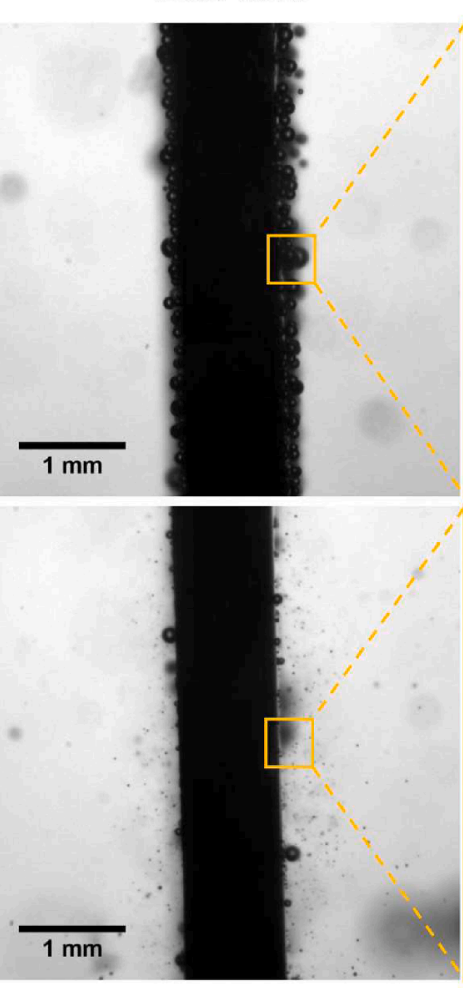

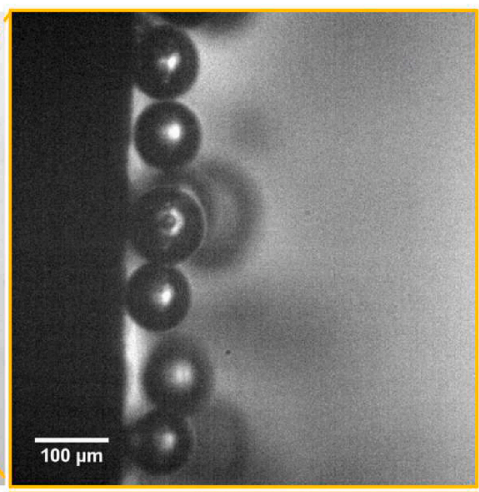

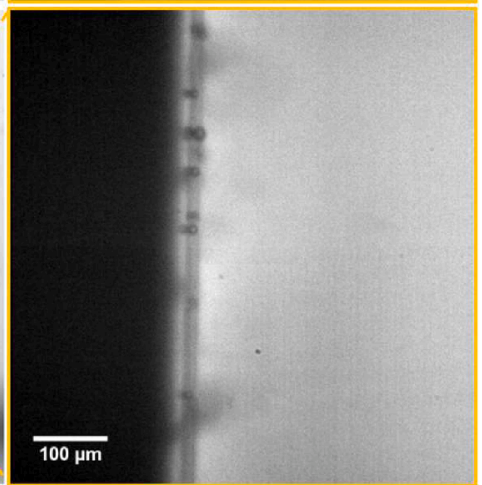

Fig. 8. Hydrogen bubbles on the surface of the stainless steel electrode under (a) silent condition and (b) sonication ( $20 \mathrm{kHz}, 100 \% \mathrm{ultrasonic}$ amplitude) at $-0.58 \mathrm{~V}$ vs. RHE in $0.5 \mathrm{M} \mathrm{NaOH}$. 
cluster. These tiny bubbles can be also generated by the collision between the bubbles growing on the electrode surface and the bubbles moving upward. When tiny bubbles on the electrode surface collided with rising bubbles without coalescence, they were bounced off. After rising bubbles had passed, the tiny bubbles were dispersed into the electrolyte (Fig. 7(b)). These bubbles dispersed by fragmentation and collision stayed near the electrode plate in the electrolyte solution. These dispersed bubbles oscillated and started to coalesce with other dispersed bubbles in the ultrasonic field (Fig. 7(c)). Then they were attracted to the bubbles attached to the electrode surface by the secondary Bjerknes force [39]. Videos A, B and C show the bubble dynamics on the electrode surface in the ultrasonic field. These videos were recorded at 5,000 fps - playback at $16 \mathrm{fps}$ (see Supplementary).

\subsection{Effect of ultrasound on bubble size and velocity}

\subsubsection{Critical diameter of bubbles in HER}

Fig. 8 shows images of the bubbles during HER on a stainless steel plate. The onset potential of $-0.58 \mathrm{~V}$ vs. RHE was applied with a current density of $30 \mathrm{~mA} / \mathrm{cm}^{2}$. Under silent condition, the electrode surface was covered with bubbles (Fig. 8(a)). In contrast, under sonication, the electrode surface area looked clean as only small bubbles were left due to fast gas removal (Fig. 8(b)). The bubbles nucleated on the electrode surface and grew during electrolysis. When the bubbles reached a critical size, they detached from the electrode surface as their buoyancy exceeded the interfacial tension between the electrode surface and the bubble, and began to rise with a uniform velocity. To measure the size of the bubbles, an image processing method using the ImageJ software program was utilized. Analyzing the high magnification images from both the front and side views, the critical diameter of bubbles under silent condition was determined to be $58.0 \mu \mathrm{m}$. The residence time of bubbles, from growth to detachment from the electrode surface, was $533 \mathrm{~ms}$. However, under continuous sonication, the bubbles could not grow steadily and were removed rapidly. The critical diameter under sonication was $15.9 \mu \mathrm{m}$ and the residence time was $118 \mathrm{~ms}$. Bubbles which reached the critical diameter left the nucleation site and started to hover around, coalescing with other bubbles on the electrode surface in the ultrasonic field.

\subsubsection{Bubble size distribution in HER}

Fig. 9 shows the histograms of the bubble size distribution in HER on a stainless steel plate under silent condition and sonication $(20 \mathrm{kHz}$, $100 \%$ ultrasonic amplitude). Image processing of the bubbles in the front view images in Fig. 8 was performed, to determine the size distribution of the bubbles. Table 1 gives the total number and the mean diameter of hydrogen bubbles under the silent and sonicated conditions. The total number of bubbles in the silent and sonicated conditions were

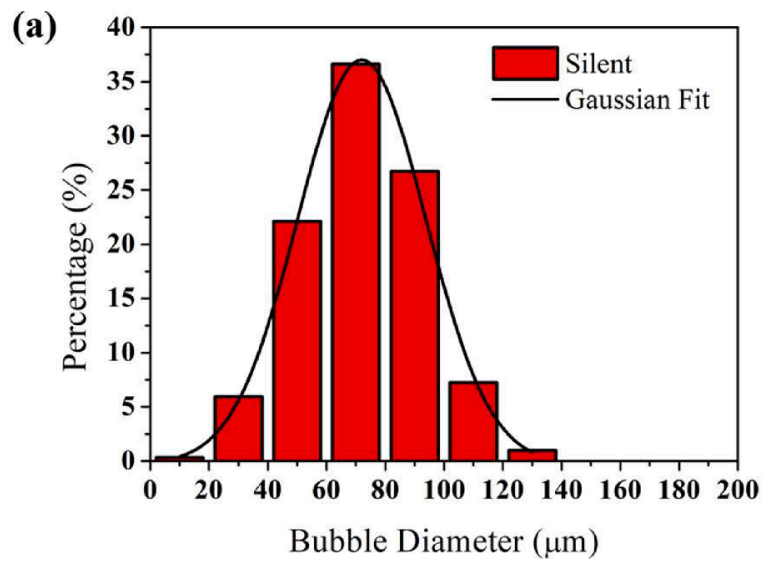

Table 1

Total number and the mean diameter of the bubbles during HER.

\begin{tabular}{llll}
\hline Condition & N total & Mean diameter $[\mu \mathrm{m}]$ & Standard deviation $[\mu \mathrm{m}]$ \\
\hline Silent & 303 & 71.8 & 20.9 \\
Sonicated & 472 & 17.0 & 12.4 \\
\hline
\end{tabular}

303 and 472, respectively. The mean diameters of the bubbles was 71.8 and $17.0 \mu \mathrm{m}$ in the silent and sonicated conditions. As previously shown in Fig. 8, the bubbles rose fast after coalescence, and only multiple small bubbles were left on the surface of the electrode after a few seconds of ultrasonic irradiation. The total number of bubbles under sonication was about 1.6 times higher than under silent condition. Especially, the quantity of small size bubbles (less than $20 \mu \mathrm{m}$ ) was larger under sonication, which increased the total bubble numbers. The bubble nucleation was more active under sonication due to increased electron mobility and enhanced mass transfer at the fluid-solid boundary layer [31]. Also, in alkaline water electrolysis under sonication, the erosion on the electrode surface occurs. Thus, bubbles can be nucleated more easily as the surface area at the nucleation site becomes larger [40].

Comparing Fig. 10(a) with Fig. 10(b), one can see that the active area exposed to the electrolyte under sonication is larger than under silent condition. The ratio of the bubble area on the electrode surface decreased from $8.3 \%$ to $1 \%$.

\subsubsection{Bubble velocity in HER}

We measured the bubble velocity with a PIV (Particle Image Velocimetry) method using PIVlab in Matlab. Fig. 10 shows the velocity vector and the magnitude of bubbles during HER on a stainless steel plate (a) under silent condition, (b) immediately after ultrasound irradiation, and (c) under continuous sonication $(20 \mathrm{kHz}, 100 \%$ ultrasonic amplitude). All of the bubbles began to rise upward after detachment from the electrode surface, and the velocity of the bubbles increased until they reached their terminal velocity. Large velocity vectors were observed on the upper side of the electrode under silent condition (Fig. 10(a)). The maximum velocity under silent condition was $0.01 \mathrm{~m} /$ s.

As the ultrasound irradiation started, bubbles stopped rising and began to move toward nearby bubbles. It was observed that most bubbles tended to move downward or sideways to coalesce with increased velocity, not upward, under sonication. The maximum velocity when ultrasound irradiation started was $0.025 \mathrm{~m} / \mathrm{s}$ (Fig. 10(b)). Under sonication, velocity magnitude increased with the size of a bubble. Under continuous sonication, the bubble reached the maximum velocity of $0.06 \mathrm{~m} / \mathrm{s}$, which was higher than the bubble velocity $(0.01 \mathrm{~m} / \mathrm{s})$ under the silent condition, although the sizes of the bubbles in both the conditions were approximately same (Fig. 10(c)). Large-sized bubbles

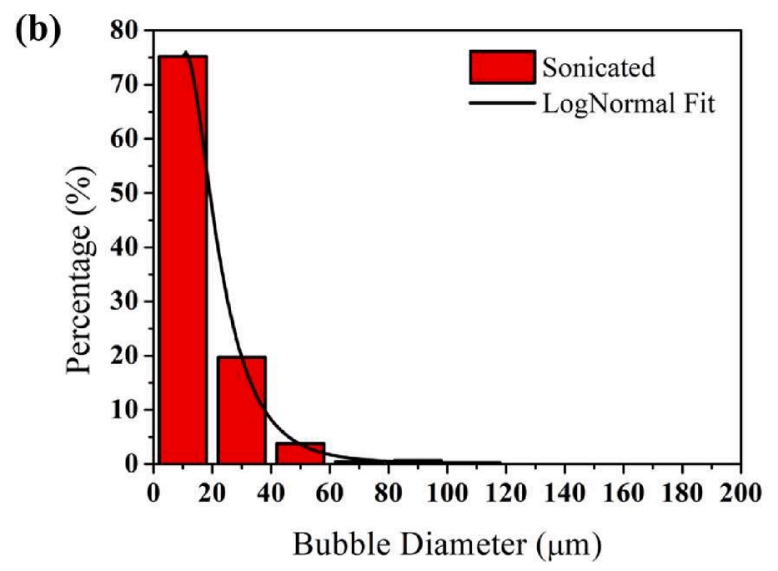

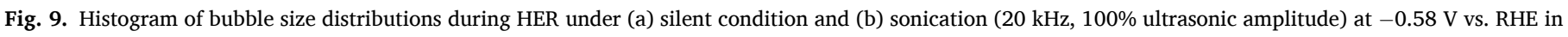
$0.5 \mathrm{M} \mathrm{NaOH}$. 
(a)

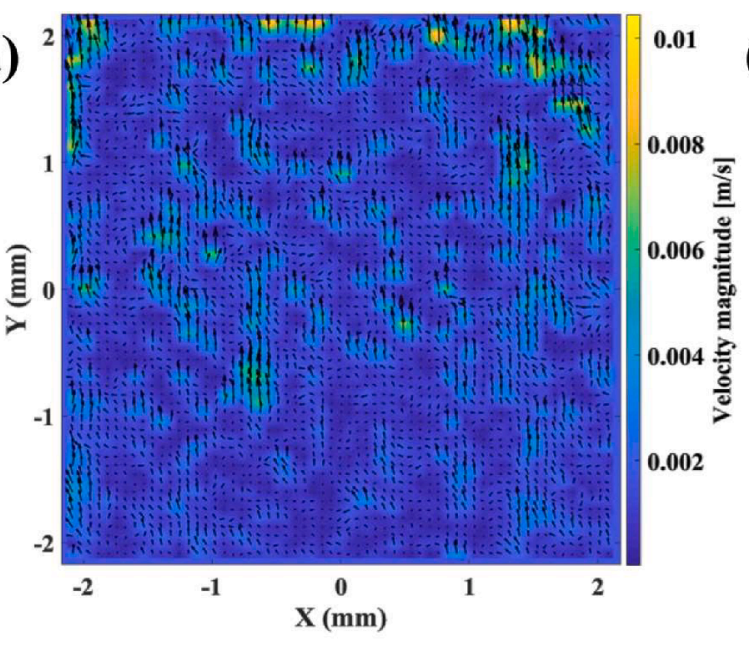

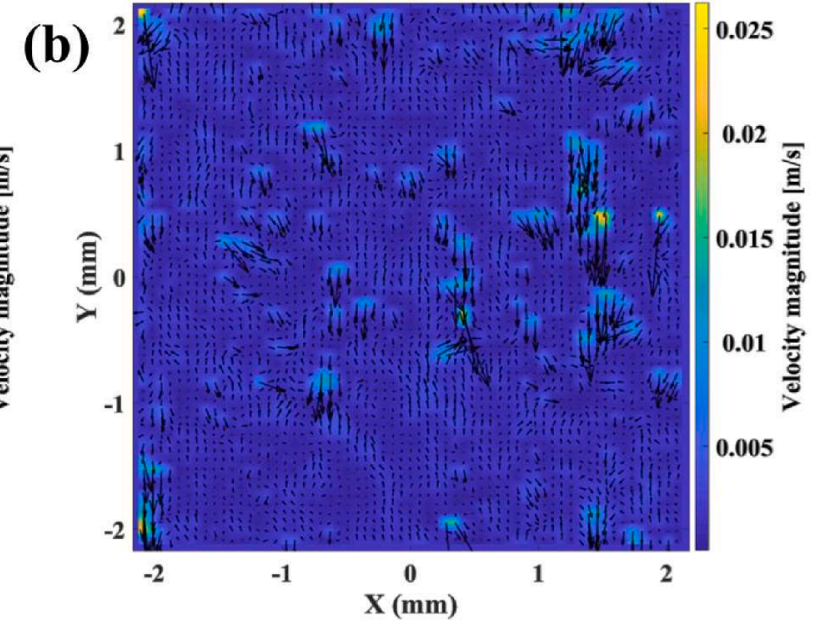

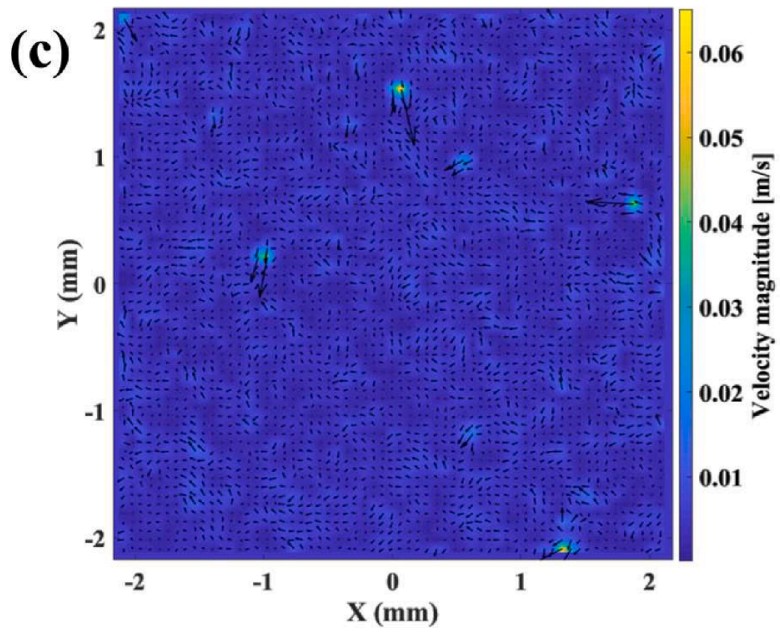

Fig. 10. Bubble velocity vectors during HER at $-0.58 \mathrm{~V}$ vs. RHE in $0.5 \mathrm{M} \mathrm{NaOH}$ : (a) under silent condition, (b) immediately after ultrasound irradiation, and (c) under continuous sonication at $20 \mathrm{kHz}$ and $100 \%$ ultrasonic amplitude.

moved even faster. Although the rising velocity of a single bubble was decreased in the bulk solution in the ultrasonic field by the Bjerknes force [41], the bubble velocity at the electrode surface was increased by coalescing into larger bubbles, and by sliding on the electrode in the ultrasonic field.

\section{Conclusions}

In this study, we investigated the effect of power ultrasound $(20 \mathrm{kHz}$, $100 \%$ ultrasonic amplitude) on HER on a stainless steel plate electrode in $0.5 \mathrm{M} \mathrm{NaOH}$ solution at $298 \mathrm{~K}$ using linear sweep voltammetry (LSV) and high-speed imaging. We found a mechanism for the hydrodynamic behavior of hydrogen bubbles on the vertical electrode plate in the ultrasonic field. In the ultrasonic field bubbles oscillated and began to agglomerate and coalesce with large bubbles nearby. These coalesced bubbles glided up and agglomerated along the surface of the vertical electrode as their buoyancy increased, but did not detach from the surface. Repeated fragmentation and coalescence of the agglomerated bubbles was observed as they were rising. Tiny bubbles were dispersed into the electrolyte near the electrode surface due to the fragmentation and collision of bubbles. These dispersed bubbles were moved back to the electrode surface by the secondary Bjerknes force. Since large bubbles were removed fast due to coalescence, only small bubbles were mainly observed on the electrode surface in the ultrasonic field. Both the critical diameter and the residence time of the bubbles on the electrode surface decreased. Also, the overall size of hydrogen bubbles on the electrode surface decreased. On the other hand, bubble nucleation was increased on the electrode surface by the improved mass transfer due to ultrasound irradiation. The bubble coverage area on the electrode surface was reduced, although the total number of hydrogen bubbles increased. As a result, the current density of the electrode increased by about 9.0, 5.9, and $2.8 \%$ in $0.1,0.5$, and $1 \mathrm{M} \mathrm{NaOH}$ electrolytes, respectively, due to fast surface cleaning. The results of this study suggest that ultrasound has a significant effect on the activation and removal of gas bubbles on the electrode surface during water electrolysis, leading to an increase in water electrolysis efficiency.

\section{CRediT authorship contribution statement}

Kyung Min Cho: Formal analysis, Visualization, Writing - original draft, Investigation, Methodology, Software. P.R. Deshmukh: Writing review \& editing, Formal analysis, Validation. Weon Gyu Shin: Conceptualization, Methodology, Visualization, Supervision, Project administration, Funding acquisition, Writing - review \& editing.

\section{Declaration of Competing Interest}

The authors declare that they have no known competing financial interests or personal relationships that could have appeared to influence the work reported in this paper. 


\section{Acknowledgements}

This work was supported by the National Research Foundation of Korea (NRF) grant funded by the Korea government (MSIT) (NRF2019R1A2C1010862).

\section{Appendix A. Supplementary data}

Supplementary data to this article can be found online at https://doi. org/10.1016/j.ultsonch.2021.105796.

\section{References}

[1] P.P. Edwards, V.L. Kuznetsov, W.I.F. David, N.P. Brandon, Hydrogen and fuel cells: Towards a sustainable energy future, Energ, Policy 36 (2008) 4356-4362.

[2] S. Merouani, O. Hamdaoui, The size of active bubbles for the production of hydrogen in sonochemical reaction field, Ultrason Sonochem 32 (2016) 320-327.

[3] J. Turner, G. Sverdrup, M.K. Mann, P.C. Maness, B. Kroposki, M. Ghirardi, R. J. Evans, D. Blake, Renewable hydrogen production, Int J Energ Res 32 (2008) 379-407.

[4] C. Coutanceau S. Baranton T. Audichon B.G. Pollet Hydrogen electrochemical production (Hydrogen and Fuel Cells Primers) 1st Edition. 2017 Academic Press ISBN: 978-0-12-811250-2.

[5] H. Nazir, C. Louis, S. Jose, J. Prakash, N. Muthuswamy, M.E.M. Buan, C. Flox, S. Chavan, X. Shi, P. Kauranen, T. Kallio, G. Maia, K. Tammeveski, N. Lymperopoulos, E. Carcadea, E. Veziroglu, A. Iranzo, A.M. Kannan, Is the H2 economy realizable in the foreseeable future? Part I: H2 production methods, Int J Hydrogen Energ 45 (27) (2020) 13777-13788.

[6] L.R.F.A.J. Bard, Electrochemical Methods: Fundamentals and Applications, John Wiley \& Sons Inc, 2001.

[7] H. Vogt, The incremental ohmic resistance caused by bubbles adhering to an electrode, Journal of Applied Electrochemistry 13 (1982) 87-88.

[8] R. Kothari, D. Buddhi, R.L. Sawhney, Studies on the effect of temperature of the electrolytes on the rate of production of hydrogen, Int J Hydrogen Energ 30 (2005) 261-263.

[9] W.W. Xu, Z.Y. Lu, X.M. Sun, L. Jiang, X. Duan, Superwetting Electrodes for GasInvolving Electrocatalysis, Accounts Chem Res 51 (2018) 1590-1598.

[10] J.J.J. Chen, K.X. Qian, J.C. Zhao, Resistance due to the presence of bubbles in an electrolytic cell with a grooved anode, Chem Eng Res Des 79 (2001) 383-388.

[11] P. Tiwari, G. Tselzouras, K. Wagner, G.F. Swiegers, G.G. Wallace, A new class of bubble-free water electrolyzer that is intrinsically highly efficient, Int J Hydrogen Energ 44 (2019) 23568-23579.

[12] M.M. Bakker, D.A. Vermaas, Gas bubble removal in alkaline water electrolysis with utilization of pressure swings, Electrochim Acta 319 (2019) 148-157.

[13] J.A. Koza, S. Muhlenhoff, P. Zabinski, P.A. Nikrityuk, K. Eckert, M. Uhlemann, A. Gebert, T. Weier, L. Schultz, S. Odenbach, Hydrogen evolution under the influence of a magnetic field, Electrochim Acta 56 (2011) 2665-2675.

[14] M.Y. Wang, Z. Wang, Z.C. Guo, Understanding of the intensified effect of super gravity on hydrogen evolution reaction, Int J Hydrogen Energ 34 (2009) 5311-5317.

[15] J. Theerthagiri, J. Madhavan, S.J. Lee, M.Y. Choi, M. Ashokkumar, B.G. Pollet, Sonoelectrochemistry for energy and environmental applications, Ultrason Sonochem 63 (2020) 104960, https://doi.org/10.1016/j.ultsonch.2020.104960.

[16] N. MORIGUCHI, The influence of supersonic waves on chemical phenomena, III The influence on the concentration polarisation, Nippon Kagaku Kaishi 55 (8) (1934) 749-750.

[17] S.-D. Li, C.-C. Wang, C.-Y. Chen, Water electrolysis in the presence of an ultrasonic field, Electrochim Acta 54 (15) (2009) 3877-3883.

[18] R.G. Compton, J.C. Eklund, F. Marken, Sonoelectrochemical processes: A review, Electroanal 9 (7) (1997) 509-522.
[19] Pollet B.G., Power Ultrasound in Electrochemistry: From Versatile Laboratory Tool to Engineering Solution, John Wiley \& Sons, Hoboken, NJ, USA, 2012.

[20] S.H. Zadeh, Hydrogen Production via Ultrasound-Aided Alkaline Water Electrolysis, Journal of Automation and Control, Engineering 2 (1) (2014) 103-109.

[21] D. Symes, Sonoelectrochemical (20 kHz) Production of Hydrogen from Aqueous Solutions, University of Birmingham, Birmingham, UK, 2011.

[22] B.G. Pollet, J.P. Lorimer, S.S. Phull, T.J. Mason, D.J. Walton, J.Y. Hihn, V. Ligier, M. Wery, The effect of ultrasonic frequency and intensity upon electrode kinetic parameters for the $\mathrm{Ag}(\mathrm{S} 2 \mathrm{O} 3)(2)(3-) / \mathrm{Ag}$ redox couple, Journal of Applied Electrochemistry 29 (1999) 1359-1366.

[23] B.G. Pollet, J.P. Lorimer, J.-Y. Hihn, S.S. Phull, T.J. Mason, D.J. Walton, The effect of ultrasound upon the oxidation of thiosulphate on stainless steel and platinum electrodes, Ultrason Sonochem 9 (5) (2002) 267-274.

[24] B.G. Pollet, J.P. Lorimer, J.-Y. Hihn, F. Touyeras, T.J. Mason, D.J. Walton, Electrochemical study of silver thiosulphate reduction in the absence and presence of ultrasound, Ultrason Sonochem 12 (1-2) (2005) 7-11.

[25] M.H. Islam, O.S. Burheim, B.G. Pollet, Sonochemical and sonoelectrochemical production of hydrogen, Ultrason Sonochem 51 (2019) 533-555.

[26] F. Foroughi, J.J. Lamb, O.S. Burheim, B.G. Pollet, Sonochemical and Sonoelectrochemical Production of Energy Materials, Catalysts 11 (2) (2021) 284, https://doi.org/10.3390/catal11020284.

[27] J. Klima, C. Bernard, Sonoassisted electrooxidative polymerisation of salicylic acid: role of acoustic streaming and microjetting, J Electroanal Chem 462 (1999) $181-186$.

[28] M.d. Hujjatul Islam, B. Naidji, L. Hallez, A. Et Taouil, J.-Y. Hihn, O.S. Burheim, B. G. Pollet, The use of non-cavitating coupling fluids for intensifying sonoelectrochemical processes, Ultrason Sonochem 66 (2020) 105087, https://doi. org/10.1016/j.ultsonch.2020.105087.

[29] T.J. Mason, The Uses of Ultrasound in Chemistry, Royal Society of Chemistry, 1990.

[30] R.F. Contamine, A.M. Wilhelm, J. Berlan, H. Delmas, Power Measurement in Sonochemistry, Ultrason Sonochem 2 (1) (1995) S43-S47.

[31] B.G. Pollet, F. Foroughi, A.Y. Faid, D.R. Emberson, M.H. Islam, Does power ultrasound $(26 \mathrm{kHz})$ affect the hydrogen evolution reaction (HER) on Pt polycrystalline electrode in a mild acidic electrolyte? Ultrason Sonochem 69 (2020).

[32] V.F.K. Bjerknes. Fields of Force, Columbia University Press, New York, 1906, pp. $1-136$.

[33] T.G. Leighton, A.J. Walton, M.J.W. Pickworth, Primary Bjerknes forces, Eur. J. Phys. 11 (1) (1990) 47-50.

[34] L.A. Crum, Bjerknes forces on bubbles in a stationary sound field, The Journal of the Acoustical Society of America 57 (1975) 1363-1370.

[35] J.G.S. Moo, C.C. Mayorga-Martinez, H. Wang, W.Z. Teo, B.H. Tan, T.D. Luong, S. R. Gonzalez-Avila, C.D. Ohl, M. Pumera, Bjerknes Forces in Motion: Long-Range Translational Motion and Chiral Directionality Switching in Bubble-Propelled Micromotors via an Ultrasonic Pathway, Adv Funct Mater 28 (2018).

[36] J.J. Jiao, Y. He, K. Yasui, S.E. Kentish, M. Ashokkurnar, R. Manasseh, J. Lee, Influence of acoustic pressure and bubble sizes on the coalescence of two contacting bubbles in an acoustic field, Ultrason Sonochem 22 (2015) 70-77.

[37] J. Ellenberger, J.M. van Baten, R. Krishna, Intensification of bubble columns by vibration excitement, Catal Today 79 (2003) 181-188.

[38] M. Postema, P. Marmottant, C.T. Lancee, S. Hilgenfeldt, N. De Jong, Ultrasound induced microbubble coalescence, Ultrasound Med Biol 30 (2004) 1337-1344.

[39] K. Yoshida, T. Fujikawa, Y. Watanabe, Experimental investigation on reversal of secondary Bjerknes force between two bubbles in ultrasonic standing wave, J Acoust Soc Am 130 (2011) 135-144.

[40] I. Hamidah, A. Solehudin, A. Setiawan, A. Hamdani, M.A.S. Hidayat, F. Adityawarman, F. Khoirunnisa, A.B.D. Nandiyanto, Corrosion study of AISI 304 on $\mathrm{KOH}, \mathrm{NaOH}$, And NaCL solution as an electrode on electrolysis process, J Eng Sci Technol 13 (2018) 1345-1351.

[41] J.M. Fan, Z. Cui, Effect of acoustic standing wave in a bubble column, Ind Eng Chem Res 44 (2005) 7010-7018. 